\title{
Suppression of Ventricular Arrhythmias After Myocardial Infarction by AT1 Receptor Blockade: Role of the AT2 Receptor and Casein Kinase 2/Kir2.1 Pathway
}

\author{
Editorial to: "Valsartan Upregulates Kir2.1 in Rats Suffering from Myocardial Infarction \\ Via Casein Kinase 2" by Xinran Li et al.
}

\author{
Bodh I. Jugdutt ${ }^{1}$
}

Published online: 15 July 2015

(C) Springer Science+Business Media New York 2015

$\begin{array}{ll}\text { Abbreviations } \\ \text { AAD } & \text { Antiarrhythmic drug } \\ \text { AngII } & \text { Angiotensin II } \\ \mathrm{AT}_{1} \mathrm{R} & \text { AngII type } 1 \text { receptor } \\ \mathrm{AT}_{2} \mathrm{R} & \text { AngII type } 2 \text { receptor } \\ \mathrm{ARB} & \mathrm{AT}_{1} \mathrm{R} \text { blocker } \\ \mathrm{CK} 2 & \text { Casein kinase } 2 \\ \mathrm{ICD} & \text { Implantable cardioverter-defibrillator } \\ \mathrm{IK} 1 & \text { Inward rectifier K+ current } \\ \mathrm{LV} & \text { Left ventricular } \\ \mathrm{MI} & \text { Myocardial infarction } \\ \mathrm{PES} & \text { Programmed electrical stimulation } \\ \mathrm{PVC} & \text { Premature ventricular complex } \\ \mathrm{RAAS} & \text { Renin-angiotensin-aldosterone system } \\ \mathrm{SCD} & \text { Sudden cardiac death } \\ \text { VAs } & \text { Ventricular arrhythmias } \\ \text { VT } & \text { Ventricular tachycardia } \\ \text { VF } & \text { Ventricular fibrillation }\end{array}$

Ventricular arrhythmias (VAs) after myocardial infarction (MI) have been a source of concern for decades because they contribute to mortality in the post-MI survivors. Common VAs, spontaneous or programmed electrical stimulation (PES) induced, include premature ventricular depolarizations or complexes (PVCs), couplets, triplets, non-sustained ven-

Bodh I. Jugdutt

bjugdutt@ualberta.ca

1 2C2 Walter MacKenzie Health Sciences Centre, Division of Cardiology, University of Alberta, Edmonton, Alberta T6G 2R7, Canada tricular tachycardia (VT) and ventricular fibrillation (VF) or VT/VF. Without doubt, major advances have been made in therapy for their control and prevention, with the development of pharmacologic and electrophysiological approaches including antiarrhythmic agents, beta-adrenergic drugs, catheter ablation, and the implantable cardioverter-defibrillator (ICD). In parallel, an added benefit of renin-angiotensin-aldosterone system (RAAS) blockers introduced for therapy of post-MI left ventricular (LV) remodeling and dysfunction, including the angiotensin II (AngII) type 1 receptor $\left(\mathrm{AT}_{1} \mathrm{R}\right)$ antagonists or blockers (ARBs), has been the suppression of VAs and sudden cardiac deaths (SCDs) as well as "final" deaths and their contribution to improved survival and outcome [1-7]. However, the precise molecular mechanism for the ARBinduced suppression of VAs has been elusive. A major goal of translational research has therefore been to not only unravel the underlying molecular mechanisms for post-MI VAs in general, but also to identify the precise molecular mechanisms and novel pathways resulting in their suppression so that they may be specifically targeted. There is a definite need for new therapies because a significant percentage of post-MI survivors continue to develop VAs resulting in SCD or "final" death despite optimal therapy including reperfusion $[4,7]$.

The electrical mechanisms of post-MI VAs have been extensively studied in experimental models since the 1980s. The traditional concept for VA induction in both MI and non-MI hearts has revolved around reentry, triggers and substrates [8-12]. Most cardiology students are familiar with the simple form of re-entry due to an anatomic obstacle described by Mines in 1913, and the classic Schmitt-Erlanger model of unidirectional block and re-entry proposed in 1929 . However, MI is rather unique in that it results in variable degrees of regional remodeling of myocardial structure, matrix, morphology, topography and electrophysiological 
properties including ionic, metabolic and electrical (Fig. 1). The marked regional heterogeneity and anisotropy promotes the vulnerable substrate for re-entry. As expected, because of the dynamic nature of the evolving MI and remodeling processes, and different degrees of heterogeneity and ischemia/ reperfusion, the substrate and mechanism of VT/VF and VA differ between acute, subacute, chronic and remote MI. In a simple construct, increased automaticity in impaired but ischemia-resistant His-Purkinje fibers and focal re-excitation, due to current flowing between myocardial fibers, result in ectopic repolarizations and triggered activity in areas of ischemia and lead to VAs during acute MI [12]. In contrast, impaired regional excitability and conductivity of ischemic zones and infarct borders (containing islands of ischemic tissue especially after coronary reperfusion), and patches of scar tissue during the subacute healing/repair phase offer the fertile arrhythmogenic substrate for reentry and sustained VA [12 for review]. Studies suggest that the dominant mechanism for VA is re-entry in ischemia and triggered activity in reperfusion
[12]. Of note, experimental studies in the 1980s established that post-MI bradycardia triggers PVCs and VT/VF in the dog model, suggesting the need for aggressive prevention and treatment of bradyarrythmias. Intramural re-entry leading to sustained rapid VT can result in circulatory collapse and shock due to just ionic and electrical remodeling $[10,12]$. Polymorphic VT that degenerates into VF can lead to SCD or "final" death. The highest risk for SCDs is during the first 30 days after MI [5].

The clinical management of MI and associated VAs has evolved dramatically since the 1980s [12-14]. Although early myocardial reperfusion reduces early mortality from VAs with sustained VT/VF and SCD [12], reperfusion itself results in profound electrophysiological remodeling with increased VT/ VF depending on the duration of prior ischemia [11] and various modulators of arrhythmogenesis (Fig. 1). Additionally, late reperfusion is associated with reperfusion damage, necrosis, and structural and matrix remodeling [15] that may lead to VAs, and late presenters after acute MI remain at increased

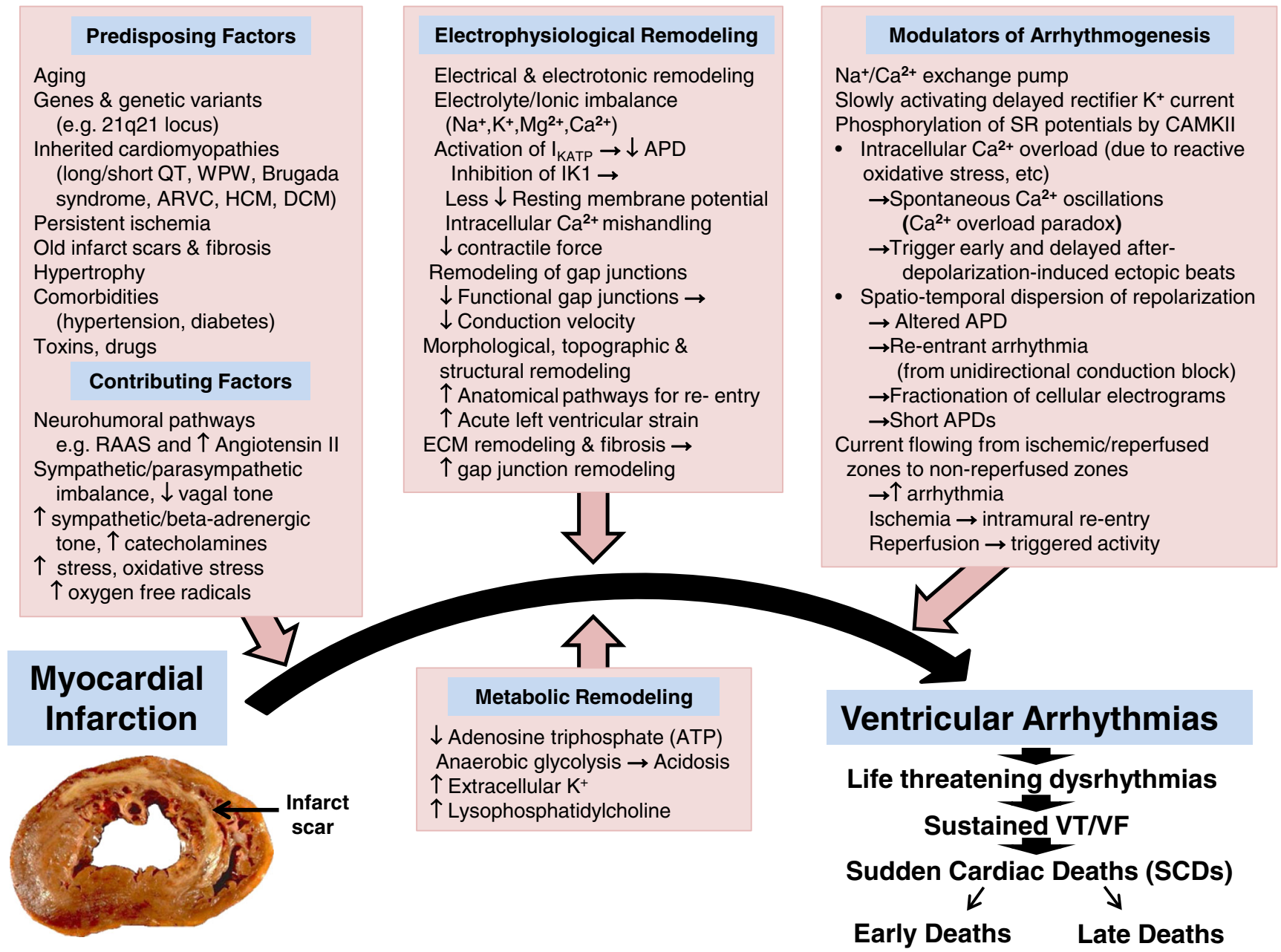

Fig. 1 Factors in genesis of ventricular arrhythmias after myocardial infarction. $\uparrow$, increase; $\downarrow$, decrease; $A P D$ action potential duration; $A R V C$ arrhythmogenic right ventricular cardiomyopathy; CAMKII calcium and calmodulin-dependent protein kinase 2; DCM dilated cardiomyopathy; ECM extracellular matrix; $S R$ sarcoplasmic reticulum; $H C M$ hypertrophic cardiomyopathy; WPW Wolf-Parkinson-White syndrome. Other abbreviations as in text 
risk of VA. Despite thrombolytic therapy in an early reperfusion trial [16], sustained VT/VF impacted negatively on outcome at 1 year; 30 -day VT/VF mortality was $44.5 \%$ and 1 year VT/VF mortality was $48.4 \%$, while 1 -year mortality of 30-day survivors was $7.1 \%$ [16]. Although revascularization procedures, infarct-limiting and anti-remodeling therapies, and beta-blockers have contributed to lower the incidence of sustained VAs within the first $48 \mathrm{~h}$ of MI, randomized trial data show that sustained VAs still develop in about $6 \%$ of patients during that interval [12]. VT/VF occurs in $\sim 6 \%$ patients undergoing primary percutaneous coronary intervention and was associated with 3-fold higher 90-day mortality, and the risk was higher late than early VT/VF [12]. Antiarrhythmic drugs (AADs) in acute MI remain controversial and their use for prophylaxis has been discouraged [12]. In the dog model, the defibrillation threshold is higher for spontaneous VF than PES-induced VF in acute MI hearts with or without reperfusion, underscoring the different metabolic or pathologic derangements in causation that need to be considered in assessing AAD therapies and designing of ICDs to control VT/VF [17].

Since the 1980s, evidence has been trickling in concerning the contributions of infarct size, infarct expansion and both infarct and structural ventricular remodeling in the acute MI phase, and further remodeling during the subsequent subacute healing/repair and chronic phases to VAs [18]. During infarct healing, focal fibrosis can either block or delay conduction; in infarct centers where damage is more homogeneous, block dominates whereas in infarct borders where damage is patchy, fibrosis widens the separation between muscle bundles and provides new, stable, anatomical channels for reentry circuits and monophasic VT given the appropriate trigger, such as sinus rate change (bradycardia or tachycardia) or PVCs. Reperfused infarcts have more patchy infarct centers and borders and spared epicardial rims, providing potentially more tracts for reentry, thereby increasing the risk for VT/VF. Infarct-limiting therapies that salvage epicardial rims may buttress against remodeling but provide the vulnerable substrate for reentry and VT/VF. Studies suggest that increased anisotropy in the epicardial border zone of healing canine infarcts contribute to reentrant circuits and VT by slowing transverse conduction due to decreased side-to-side coupling between myocytes independent of connexin43 gap junctional protein expression [19]. Post-MI survivors remain at risk for monophasic VT, but this risk (or that of sustained spontaneous or inducible VT) has decreased in those receiving recommended therapies, including $\mathrm{AT}_{1} \mathrm{R}$ antagonists or $\mathrm{ARBs}$ as well as ICDs for recurrent VT and catheter ablation in those refractory to AADs. However, the 5-year mortality risk is still significant, and the risk remains high in patients with large MIs or unsuccessfully reperfused MIs.

Studies of molecular correlates of potassium current remodeling underscored the multiple cellular and molecular mechanisms underlying the downregulation of potassium currents in failing hearts [10]. For example in the rapid-pacinginduced heart failure rabbit model, reduced inward-rectifier $\mathrm{K}^{+}$(IK1) current occurs without decrease in Kir2.1 mRNA level, implying that the reduced current results from posttranslational modification of the channel protein [10]. In the rapid-pacing-induced heart failure dog model, moderate myocardial dysfunction was associated with significant electrophysiological abnormalities and VF, but no changes in myocardial cell morphology or intercellular connections, or expression of gap junction proteins connexin 43 and connexin 45 , suggesting a dominant functional abnormality in cell-to-cell communication [20]. In the isolated perfused dog heart, spatiotemporal resolution mapping of optical transmembrane potentials detected erupting rotors that emit reentrant waves during early VF, and "evolved" or deteriorated into a pattern lacking the spatiotemporal features of epicardial rotors [21]. Whether such mapping may capture chaotic impulse propagation in the heterogeneous substrate of MIs and transition to more chaotic VT/VF and contribute to discovery and development of new therapies for post-MI VAs needs study.

Various mechanisms for the suppression of post-MI VAs by RAAS blockade including $\mathrm{AT}_{1} \mathrm{R}$ antagonists such as valsartan have been postulated (Fig. 2). Increased AngII after $\mathrm{MI}$ and the arrhythmogenic effect of AngII acting via $\mathrm{AT}_{1} \mathrm{R}$ are well documented [6, 22-24]. The postulated mechanisms have centered around limitations of infarct size, adverse postMI infarct zone and structural LV remodeling $[6,18,24]$ and electrical remodeling [1-7]. Recent experimental studies with valsartan post $\mathrm{MI}$ documented decreased $\mathrm{AT}_{1} \mathrm{R}$ expression, decreased fibrosis and restored connexin 43 in the border zone and reduced PES-induced VAs [25], and reduced transmural dispersion of repolarization and electrical heterogeneity with preserved the density of the outward potassium current (Ito) [26]. In this issue of Cardiovascular Drugs and Therapyl, Li et al. explored the effect of valsartan on the suppression of casein kinase 2 (CK2) and remodeling of the inward rectifier potassium current (IK1) via Kir2.1 protein after MI in the rat model [27]. They treated young Wistar rats with valsartan or saline for 7 days between day 1 and day 8 after MI, and used standard Western blot and PCR techniques to measure regional myocardial CK2 and Kir2.1 protein and mRNA levels, respectively. They also overexpressed CK2 in neonatal rat ventricular myocytes by transfection using the $\mathrm{H} 9 \mathrm{c} 2$ rat embryonic ventricle cell line. They performed 6 sets of experiments in logical sequence and report 6 main findings. First, increased CK2 expression in the infarct border was associated with reduced IK1/Kir2.1 protein levels. Second, CK2 overexpression suppressed the KCNJ2/Kir2.1 expression. Third, CK2 inhibition enhanced KCNJ2/Kir2.1 expression. Fourth, compared to saline controls in vivo, valsartan reduced CK2 and increased Kir2.1 expression post MI. Fifth, hypoxia increased CK2 expression whereas valsartan inhibited CK2 


\author{
Clinical studies \\ $\downarrow$ Total \& arrhythmic mortality \\ $\downarrow$ Early VT/VF \& 30-day mortality \\ $\downarrow$ SCDs \\ $\downarrow$ Late potentials on signal \\ averaged ECGs at 6-30 days \\ Experimental studies \\ $\downarrow$ PES-induced VA \& fibrosis; \\ $\uparrow$ connexin43 \\ Reverse increase in transmural \\ dispersion of repolarization / \\ abnormal ionic flux during plateau of \\ action potential \\ Restore density of outward $\mathrm{K}^{+}$current \\ (Ito) \\ $\downarrow$ anisotropy or electrical heterogeneity \\ $\downarrow$ myocardial nor-epinephrine, \\ aldosterone, Angll \& $\mathrm{AT}_{1} \mathrm{R}$ \\ $\downarrow$ CK2 activation \\ $\uparrow$ Kir2.1 \\ Improve IK1 remodeling \\ $\mathrm{AT}_{2} \mathrm{R}$ activation during $A T_{1} \mathrm{R}$ blockade
}

ANGIOTENSINOGEN

ANGIOTENSIN I

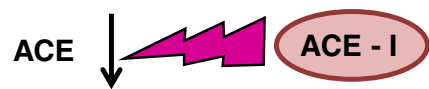

ANGIOTENSIN II

ALDOSTERONE

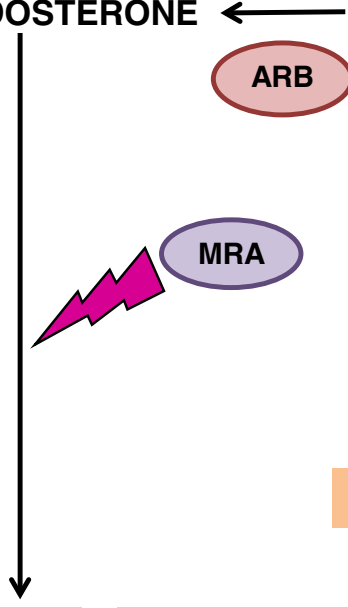

Arrhythmogenic

Salt/water retention

Inflammation

Proliferation

Fibrosis

Vascular remodeling

Ventricular remodeling

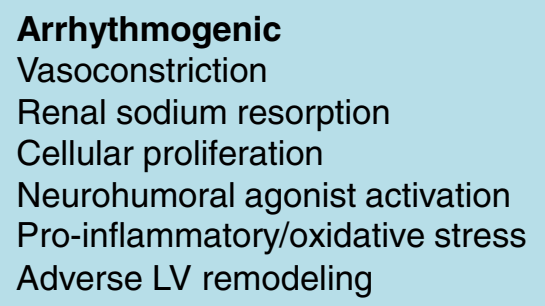

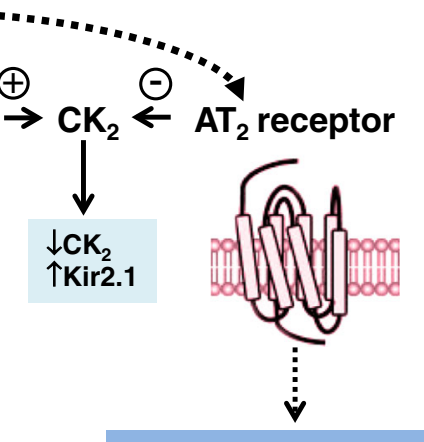

CARDIOTOXIC

$\downarrow$
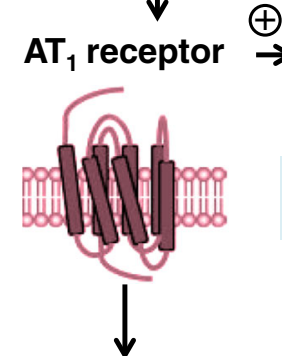

CARDIOPROTECTIVE

$\vdots$

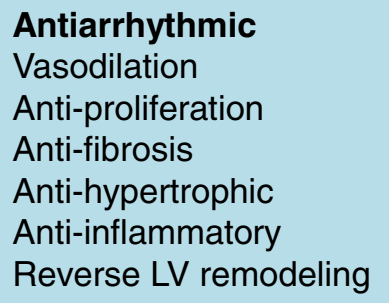

Fig. 2 Mechanisms of suppression ventricular arrhythmias after myocardial infarction by $\mathrm{AT}_{1} \mathrm{R}$ blockade and other RAAS antagonists. Enzymatic cascades and key receptor activation pathways in the RAAS with the proposed new $\mathrm{AT}_{2} \mathrm{R} / \mathrm{CK} 2 / \mathrm{Kir} 2.1$ pathway. $\uparrow$, increase; $\downarrow$,

expression in vitro. Sixth, CK2 overexpression in valsartantreated neonatal rat ventricular myocytes abolished its beneficial effect on KCNJ2/Kir2.1 in those cells. Together, these findings suggested that valsartan reduces CK2 activation, increases Kir2.1 expression and modifies IK1 remodeling after $\mathrm{MI}$ in the rat model.

The new finding that $\mathrm{AT}_{1} \mathrm{R}$ blockade after MI may contribute to reduced susceptibility to VAs during the healing/repair phase by modulation of IK1 remodeling via the CK2/Kir2.1 pathway in that study [27] has at least six important implications. First, it provides supportive and incremental data on the important topic of modification of electrical remodeling and suppression of VAs after MI by $\mathrm{AT}_{1} \mathrm{R}$ blockade. Second, it endorses the idea of an arrythmogenic effect of increased AngII during healing after MI. Third, it provides an additional explanation and new molecular mechanism for the beneficial effect of $\mathrm{AT}_{1} \mathrm{R}$ blockade on reducing susceptibility to VAs during healing after MI. Fourth, it supports the use of $\mathrm{AT}_{1} \mathrm{R}$ blocker therapy for suppressing post-MI VAs after MI and improve survival with a favorable outcome. Fifth, the CK2/ Kir2.1 pathway per se may provide a potential therapeutic decrease; $A C E$ angiotensin-converting enzyme; $A C E-I$ ACE-inhibitor; Ang angiotensin; $A T_{1} R$ and $A T_{2} R$ Ang-II type 1 and type 2 receptor, respectively; $A R B$ angiotensin receptor blocker; $M R A$ mineralocorticoid receptor antagonist. Other abbreviations as in text

target for suppressing post-MI VAs. Sixth, the discovery of this new molecular mechanism of arrhythmia suppression after MI involving the RAAS underscores the need for research into other molecular mechanisms and potential targets.

The mechanism for the $\mathrm{AT}_{1} \mathrm{R}$ blocker induced inhibition of CK2 expression deserves comment. It is known that $\mathrm{AT}_{2} \mathrm{R}$ plays a counterregulatory role opposing $\mathrm{AT}_{1} \mathrm{R}$ functions, as in the case of enhanced vasodilation via unopposed $\mathrm{AT}_{2} \mathrm{R}$ activation and downstream $\mathrm{AT}_{2}$-mediated signaling [28]. A recent editorial suggested that CK2 may be activated by $\mathrm{AT}_{1} \mathrm{R}$ and inactivated by $\mathrm{AT}_{2} \mathrm{R}$-activated SHP-1 [29] in the context of Rho inhibition via $\mathrm{AT}_{2} \mathrm{R}$ resulting in vasodilation [30]. Another report suggested that AngII induces CK2- $\alpha$ dependent kinase activity in the context of cardiomyocyte hypertrophy [31]. Also in a hypertrophy model, AngII was shown to differentially inhibit inward rectifier $\mathrm{K}^{+}$(Kir) channels in coronary vascular smooth muscle cells associated with increased $\mathrm{AT}_{1} \mathrm{R}$ but not $\mathrm{AT}_{2} \mathrm{R}$ [32]. Here, the provocative suggestion that $\mathrm{CK} 2$ is activated by $\mathrm{AT}_{1} \mathrm{R}$ and inactivated by $\mathrm{AT}_{2} \mathrm{R}$ [27] further expands the counterregulatory role of $\mathrm{AT}_{2} \mathrm{R}$ in $\mathrm{AT}_{1} \mathrm{R}$ functions (Fig. 2). $\mathrm{AT}_{1} \mathrm{R}$ blockade with unopposed or 
enhanced $\mathrm{AT}_{2} \mathrm{R}$ activation appears to have resulted in net CK2 downregulation and upregulation of Kir2.1, but evidence for this putative $\mathrm{AT}_{2} \mathrm{R} / \mathrm{CK} 2 / \mathrm{Kir} 2.1$ signaling pathway needs study.

Every study has its limitations. Here, establishment of cause and effect still remains to be shown using genetic models as mentioned by the authors [27]. Clear demonstrations of reduced VAs in vivo need to be shown with research translation in mind. Confirmation in large animal models is also needed. Since the animals were young, confirmation in older animals is needed because significant remodeling of the RAAS and other pathways occur with aging [24] and may involve post-MI electrical remodeling as well. There is extensive evidence that significant structural remodeling occurs during the acute as well as the subacute healing/repair phase after MI depending on infarct size as well as adequacy of healing/repair. Since this study really focused on the effect of $\mathrm{AT}_{1} \mathrm{R}$ blockade during the dynamic subacute healing/ repair phase, when significant structural infarct and noninfarct remodeling are known to occur with regional infarct expansion and global LV dilation/dysfunction (dependent on infarct size), beneficial effects on infarct size and structural remodeling may have contributed to reduced electrical remodeling. However, chronic and remote post-MI phases were also not studied. Finally, measurements of KCNJ2/Kir2.1 expression were not made during the acute or subacute phases of MI on myocytes from survivors of earlier electrical remodeling. Both spatial and temporal factors should be considered in post-MI electrical remodeling.

In conclusion, the finding that AT1 blockade during the healing/repair phase after MI reduces CK2 activation, which in turn increases Kir2.1 expression and ameliorates IK1 remodeling and thereby contributes to reduced susceptibility to VAs in this study [27] is important and deserves confirmation. The $\mathrm{AT}_{2} \mathrm{R} / \mathrm{CK} 2 / \mathrm{Kir} 2.1$ and $\mathrm{CK} 2 / \mathrm{Kir} 2.1$ pathways may be potential therapeutic targets for suppression of VAs post-MI.

Acknowledgments I am indebted to Bernadine A. Jugdutt for expert assistance with the figures.

Conflict of Interest None.

\section{References}

1. Budaj A, Cybulski J, Cedro K, et al. Effects of captopril on ventricular arrhythmias in the early and late phase of suspected acute myocardial infarction. Randomized, placebo-controlled substudy of ISIS-4. Eur Heart J. 1996;17:1506-10.

2. Domanski MJ, Exner DV, Borkowf CB, et al. Effect of angiotensin converting enzyme inhibition on sudden cardiac death in patients following acute myocardial infarction. A meta-analysis of randomized clinical trials. J Am Coll Cardiol. 1999;33:598-604.
3. Chiladakis JA, Karapanos D, Agelopoulos G, et al. Effects of early captopril therapy after myocardial infarction on the incidence of late potentials. Clin Cardiol. 2000;23:96-102.

4. St John Sutton M, Lee D, Rouleau JL, et al. Left ventricular remodeling and ventricular arrhythmias after myocardial infarction. Circulation. 2003;107:2577-82.

5. Solomon SA, Wang D, Finn P, et al. Effect of candesartan on causespecific mortality in heart failure patients. The Candesartan in Heart Failure Assessment of Reduction in Mortality and morbidity (CHARM) Program. Circulation. 2004;110:2180-3.

6. Jugdutt BI. Valsartan in the treatment of heart attack survivors. Vasc Health Risk Manag. 2006;2:125-38.

7. Askari AT, Shishehbor MH, Kaminski MA, on behalf of the GUSTO-V Investigators, et al. The association between early ventricular arrhythmias, renin-angiotensin-aldosterone system antagonism, and mortality in patients with ST-segment-elevation myocardial infarction: insights from global use of strategies to open coronary arteries (GUSTO) V. Am Heart J. 2009;158:238-43.

8. Janse MJ, Wit AL. Electrophysiological mechanisms of ventricular arrhythmias resulting from myocardial ischemia and infarction. Physiol Rev. 1989;69:1049-169.

9. Carmeliet E. Cardiac ionic currents and acute ischemia: from channels to arrhythmias. Physiol Rev. 1999;79:917-1017.

10. Rose J, Armoundas AA, Tian Y, et al. Molecular correlates of altered expression of potassium currents in failing rabbit myocardium. Am J Physiol Heart Circ Physiol. 2005;288:H2077-87.

11. Cherry MC, Fenton FH, Gilmour Jr RF. Mechanisms of ventricular arrhythmias: a dynamical systems-based perspective. Am J Physiol. 2012;302:H2451-63.

12. Gorenek B, Lundqvist CB, Terradellas J, et al. A. Cardiac arrhythmias in acute coronary syndromes: position paper from the joint EHRA, ACCA, and EAPCI task force. Europace. 2014;16:165573.

13. Zipes DP, et al. For European Heart Rhythm Association, Heart Rhythm Society, ACC/AHA/ESC 2006 guidelines for management of patients with ventricular arrhythmias and the prevention of sudden cardiac death: a report of the American College of Cardiology/ American Heart Association Task Force and the European Society of Cardiology Committee for Practice Guidelines (Writing committee to develop guidelines for management of patients with ventricular arrhythmias and the prevention of sudden cardiac death). Eurospace 2006;8:746-817.

14. Kusumoto FM, Calkins H, Boehmer J, et al. For HRS/ACC/AHA expert consensus statement on the use of implantable cardioverterdefibrillator therapy in patients who are not included or not well represented in clinical trials. Circulation. 2014;130:94-125.

15. Manhenke C, Ueland T, Jugdutt BI, et al. The relationship between markers of extracellular cardiacmatrix turnover. Infarct healing and left ventricular remodelling following primary PCI in patients with first-time STEMI. Eur Heart J. 2014;35:395-402.

16. Newby KH, Thompson T, Stebbins A, et al. Sustained ventricular arrhythmias in patients receiving thrombolytic therapy: incidence and outcomes. The GUSTO Investigators. Circulation. 1998;98: 2567-73.

17. Ouyang P, Brinker JA, Bulkley BH, et al. Ischemic ventricular fibrillation: the importance of being spontaneous. Am J Cardiol. 1981:48:455-9.

18. Jugdutt BI. Ventricular remodeling post-infarction and the extracellular collagen matrix. When is enough enough? Circulation. 2003;108:1395-403.

19. Yao JA, Hussain W, Patel P, et al. Remodeling of gap junctional channel function in epicardial border zone of healing canine infarcts. Circ Res. 2003;92:437-43.

20. Kavanagh KM, Guerrero PA, Jugdutt BI, et al. Electrophysiologic properties and ventricular fibrillation in normal and myopathic hearts. Can J Physiol Pharmacol. 1999;77:510-9. 
21. Witkowski FX, Leon LJ, Penkoske PA, et al. Spatiotemporal evolution of ventricular fibrillation. Nature. 1998;392:78-82.

22. de Gasparo M, Catt KJ, Inagami T, et al. International union of pharmacology. XXIII. The angiotensin II receptors. Pharmacol Rev. 2000;52:415-72.

23. Lefroy DC, Wharton J, Crake T, et al. Regional changes in angiotensin II receptor density after experimental myocardial infarction. J Mol Cell Cardiol. 1996;28:429-40.

24. Jugdutt BI. Aging and remodeling of the RAS and RAAS and related pathways: implications for heart failure therapy. In: Jugdutt BI, Editor. Aging and heart failure: mechanisms and management. Springer; 2014, pp 259-89.

25. Jiao K, Li Y, Zhang P, et al. Effects of valsartan on ventricular arrhythmia induced by programmed electrical stimulation in rats with myocardial infarction. J Cell Mol Med. 2012;16:1342-51.

26. Zhong Y, Cao C, Tong C, et al. Effect of valsartan on action potential and potassium efflux in rabbits with myocardial infarction. Afr $\mathrm{J}$ Pharm Pharmacol. 2012;6:2049-54.
27. Li X, Hesheng H, Wang Y, et al. Valsartan upregulates Kir2.1 in rats suffering from myocardial infarction via casein kinase 2 . Cardiovasc Drugs Ther. 2015;29: this issue.

28. Jugdutt BI. Expanding saga of the renin-angiotensin system. The angiotensin II counter-regulatory AT2 receptor pathway. Circulation. 2015;131:1380-3.

29. Eguchi S. Triple twist theory of Rho inhibition by angiotensin II type 2 receptor. Circ Res. 2008;102:1143-5.

30. Guilluy C, Rolli-Derkinderen M, Loufrani L, et al. Ste20-related kinase SLK phosphorylates Ser188 of RhoA to induce vasodilation in response to angiotensin II type 2 receptor activation. Circ Res. 2008;102:1265-74.

31. Hauck L, Harms C, An J, et al. Protein kinase CK2 links extracellular growth factor signaling with the control of p27(Kip1) stability in the heart. Nat Med. 2008;14:315-24.

32. Park WS, Ko J, Kim N, et al. Increased inhibition of inward rectifier $\mathrm{K}^{+}$channels by angiotensin II in small-diameter coronary artery of isoproterenol-induced hypertrophied model. Arterioscler Thromb Vasc Biol. 2007;27:1768-75. 